\title{
Putative roles of circulating resistin in patients with asthma, COPD and cigarette smokers
}

\author{
Sana S. Al Mutairi ${ }^{\mathrm{a}, *}$, Olusegun A. Mojiminiyi ${ }^{\mathrm{b}}$, Aida Shihab-Eldeen ${ }^{\mathrm{c}}$, Tahani Al Rammah ${ }^{\mathrm{b}}$ and \\ Nabila Abdella ${ }^{\mathrm{a}}$ \\ ${ }^{a}$ Department of Medicine, Faculty of Medicine, Kuwait University, Safat, Kuwait \\ ${ }^{\mathrm{b}}$ Department of Pathology, Faculty of Medicine, Kuwait University, Safat, Kuwait \\ ${ }^{\mathrm{c}}$ Department of Pharmacology, Faculty of Medicine, Kuwait University, Safat, Kuwait
}

\begin{abstract}
Aims: To investigate the hypothesis that circulating resistin reflects the degree of pulmonary inflammation, this study explores putative roles of resistin in patients with acute and stable inflammatory obstructive airway diseases and cigarette smokers. Methods: We determined complements C3, C4, fasting resistin, insulin, glucose and lipid profile; calculated insulin resistance (homeostasis model assessment (HOMA-IR) in patients with acute asthma exacerbation $(n=34)$; stable asthma $(n=26)$ and stable chronic obstructive pulmonary disease (COPD; $n=26)$, cigarette smokers $(n=81)$, and healthy control subjects $(n=$ 42). We determined the associations between these variables and pulmonary function tests.

Results: Patients with COPD, acute and stable asthma had significantly higher resistin and insulin than control subjects. Resistin, insulin, HOMA-IR, FEV1\% and FEV1/FVC were significantly $(p<0.05)$ different between patients with acute asthma compared with stable asthma and COPD; smokers had similar levels of resistin, C3 and C4 as patients with asthma and COPD. In smokers, patients with asthma or COPD, resistin showed significant inverse correlations with FEV1\%; FEV1/FVC\% and positive significant correlations with BMI and HOMA-IR. Logistic regression showed that resistin is associated $(p<0.05)$ with inflammatory obstructive airways disease - odds ratio $(\mathrm{OR})=1.22$ and smoking $\mathrm{OR}=1.18$.

Conclusion: Resistin may be a disease activity marker and may contribute to insulin resistance in smokers, asthma and COPD.
\end{abstract}

Keywords: Asthma, chronic obstructive pulmonary diseases, insulin resistance, resistin, smokers

\section{Introduction}

Resistin, also called adipose tissue-specific secretory factor (ADSF) or Found in Inflammatory Zone (FIZZ3) is a hormone, which is secreted by adipose tissue cells in mouse [1] but has been shown to be produced by monocytes with increased expression as the monocytes mature into macrophages in humans [2]. Although results have been controversial, studies on resistin have focused mainly on its potential role in obesity-related insulin resistance with studies showing lack of association $[3,4]$ and others showing significant associa-

* Address for correspondence: Dr. SS Al Mutairi, Department of Medicine, Faculty of Medicine, PO Box 24923, Safat, Kuwait, Code 13110. Tel.: 965 25319596; Fax: 965 25338907; E-mail: san.mut@ hsc.edu.kw. tions between resistin, insulin resistance and Type 2 diabetes [5,6].

Much less controversial is the association of resistin with inflammatory conditions and several studies have shown association of resistin with inflammatory factors such as TNF $\alpha$, IL-6 and C- reactive protein (CRP) [6-8]. Resistin has been linked to several inflammation-related diseases like atherosclerosis, arthritis [9] and recently to inflammatory lung diseases $[10,11]$. Larochelle et al. found patients with asthma to have higher levels of resistin compared to normal controls and those levels were increased with disease severity in an asthmatic cohort [10].

Some published studies have shown that resistin's association with inflammatory markers appeared independent of Body Mass Index (BMI) [12] though other studies showed significant BMI-dependent association with insulin resistance and factors linked with obesity 
and inflammation in patients with type 2 diabetes [6, 13]. Hypoxia, especially in the expanding adiposetissue of obese subjects, has been shown to cause dysregulated production of adipokines and could affect resistin levels $[14,15]$. In patients with obstructive inflammatory airway disease, it is unclear whether the determinants of circulating resistin are obesity related factors such as insulin resistance or the degree of inflammation. Therefore, the present study was designed to test the hypothesis that circulating resistin reflects the degree of pulmonary inflammation in patients with acute and stable inflammatory obstructive airway disease (asthma and chronic obstructive pulmonary disease (COPD) as well as subjects who smoke cigarettes and, therefore, likely to have airway inflammation. The study also aimed at evaluating the determinants and associations of resistin as well as complements C3 and $\mathrm{C} 4$ which have been shown to play key roles in the induction or amplification of airways inflammation in airways diseases such as asthma and chronic obstructive airways disease [16-18].

\section{Patients, materials and methods}

\subsection{Subjects}

Five main groups of patients were recruited for this study - group 1 subjects with acute asthma exacerbation $(n=26)$, group 2 subjects with stable chronic asthma $(n=34)$, group 3 consisted of patients with stable COPD $(n=26)$ and group 4 consisted of subjects who smoke $(n=81$; consisting of 42 subjects who smoke cigarettes and 39 subjects who smoke Sheesha (hubble-bubble or hooka - a traditional method of smoking tobacco leaves in the Middle East and Indian subcontinent) and group 5 were apparently healthy non-smoking subjects $(n=42)$. Acute and stable asthma were defined and managed according to the National Heart, Lung and Blood Institute Expert Panel Report 3 (EPR 3): Guidelines for the Diagnosis and Management of Asthma. NIH Publication number 08-4051, 2007. [19]. The COPD patients were identified by using the NHLBI/WHO Global Initiative for chronic obstructive lung disease (GOLD) Workshop Summary [20]. The subjects were interviewed by a trained nurse or a physician and details of current and past medical histories, drug history and nature of therapy and smoking status were recorded. Patients with stable asthma were maintained on low to moderate dose inhaled steroid (beclomethasone or budesonide up to $200 \mu \mathrm{g} /$ day increasing to moderate dose of up to $400 \mu \mathrm{g} / \mathrm{day}$; alternatively, some patients were maintained on fluticasone $250 \mu \mathrm{g} /$ day increasing to $500 \mu \mathrm{g} /$ day as required) plus long acting inhaled bronchodilators when indicated. While COPD patients were mainly on short or long acting bronchodilators. Severely hypoxic patients requiring mechanical ventilation were excluded from the study. In groups 1-3 and control subjects other exclusion criteria included smoking, use of oral steroids or statins and presence of any other concurrent inflammatory disease at the time of recruitment for the study. Six of the patients in group 3 had never smoked and all the other patients were ex smokers. Group 4 subjects were apparently healthy subjects who smoke and who are not on any medication. All the participating subjects gave informed voluntary consent to participate in the study according to the protocol approved by the local ethics committee and in accordance with the ethical standards of the Helsinki declaration.

Fasting (defined as eight hours or longer since the last meal before specimen collection) blood samples were collected from all participating subjects. Patients with asthma exacerbation had their blood samples collected before commencement of therapy. All anthropometric measurements were made by trained observers using standard techniques (World Health Organisation. Measuring obesity: classification and distribution of anthropometric data. Copenhagen: WHO, 1989. (Nutr UD, EUR/ICP/NUT 125) [21] with the participant wearing light clothes without shoes. Height (to the nearest $0.1 \mathrm{~cm}$ ) was determined by use of a stadiometer, and weight (to the nearest $0.1 \mathrm{~kg}$ ) was determined by use of a standardized standing beam balance and from these the body mass index (BMI) was calculated as weight $(\mathrm{kg})$ divided by the height $(\mathrm{m})$ squared.

\subsection{Spirometry}

Spirometry (Using the Jaeger Masterlab (version 4.34, GmbH, Germany) was done on all patients with asthma (acute and stable), COPD as well as cigarette smokers. The system was calibrated for body temperature and pressure of saturated gas and volume and as per American Thoracic Society Standards (ATS) [22].

\section{Laboratory methods}

\subsection{Inflammatory markers}

Levels of complement C3 and C4 were assayed on the Beckman IMMAGE ${ }^{\circledR}$ (Beckman Corporation, Brea, CA, USA) immunochemistry analyser. 


\subsection{Resistin}

Fasting plasma resistin was measured using an enzyme-linked immunoassay (ELISA) kit (BioVendor, Brno, Czech Republic) with a limit of detection of $0.20 \mathrm{ng} / \mathrm{ml}$. The inter- and intra-assay coefficients of variation on pooled plasma specimen with resistin concentration of $6.8 \mathrm{ng} / \mathrm{mL}$ were $3.4 \%$ and $5.2 \%$ respectively.

\subsection{Other assays}

Fasting plasma glucose (FPG), total cholesterol (TC), triglycerides (TG), high density lipoprotein cholesterol (HDL-C), and creatinine were analyzed on an automated analyzer (Beckman LX20, Beckman Corporation, Brea, CA, USA). The low density lipoprotein cholesterol (LDL-C) was calculated using the Friedewald formula [23].

Fasting serum insulin was determined with the Immulite 1000 automated immunoassay system (DPC, Los Angeles, USA). Insulin resistance was calculated using the homeostasis model assessment (HOMA) formula using a calculator downloaded from http://www. dtu.ox.ac.uk/index.html?maindoc=/publications/ [24]. The HOMA calculator also gives estimates of steady state beta cell function $(\% \mathrm{~B})$ and insulin sensitivity $(\% \mathrm{~S})$.

\subsection{Statistical methods}

Statistical analyses were performed using the Statistical Package for Social Sciences version 14 for windows (SPSS Inc. Chicago, USA). Distributions of continuous variables were tested for normality. Relationships between continuous variables were assessed using Pearson correlation coefficient. Study population characteristics and anthropometric and laboratory measurements were presented as mean and 95\% confidence intervals (CI). Comparison between two groups was performed with the unpaired student $t$-test and the Kruskal-Wallis analysis of variance was used to determine the differences between more than two groups. The associations of resistin with obstructive airways disease, insulin resistance and smoking were examined by binary logistic regression analysis that included age, BMI, glucose, insulin and HOMA-IR as potential confounders. Values of $p \leqslant 0.05$ were considered to be statistically significant.

\section{Results}

\subsection{General}

Table 1 summarises the results from the various study groups. The subjects were matched for BMI and fasting glucose but patients with COPD were older than control subjects because, for logistic reasons and local factors, we were unable to recruit older age-matched healthy controls. Patients with acute and stable asthma and those with COPD had significantly higher resistin and insulin than control subjects (Fig. 1). Kruskal-Wallis analysis of variance shows that resistin $(p=0.019)$, insulin $(p=0.03)$, HOMA-IR $(p=0.02)$, FEV $1 \%$ $(p=002)$ and FEV1/FVC $(p<0.0001)$ were significantly different when patients with acute asthma were compared to those with stable asthma and COPD but the inflammatory markers C3 and C4 were not. Subjects who are smokers had similar levels of the inflammatory markers $\mathrm{C} 3$ and $\mathrm{C} 4$ with patients with asthma and COPD and these were significantly higher than in control subjects.

\subsection{Correlations of resistin}

In control subjects, resistin showed modest correlation with BMI $(r=0.27 ; p=0.045)$ but did not show significant correlations with insulin, HOMA-IR and other metabolic variables.

In patients with asthma or COPD, resistin showed significant inverse correlations with FEV1\% $(r=$ $-0.41 ; p=0.01)$ and FEV1/FVC $\%(r=-0.33 ; p=$ 0.04). Resistin also showed significant correlations with BMI ( $r=0.31 ; p=0.046)$; insulin $(r=0.31$, $p=0.03)$ HOMA-IR $(r=0.42, p=027)$ and C3 ( $r=$ $0.32, p=0.046$ ) but did not show significant correlations with complement $\mathrm{C} 4$ and glucose. However, in this group, BMI also showed significant correlations with C3 $(r=0.33 ; p=0.008)$; insulin $(r=0.51$; $p<0.0001)$; glucose $(r=0.28 ; p=0.028)$. Figure 2 shows the correlation of resistin with $\mathrm{C} 3$ in patients with acute asthma.

The mean $(95 \% \mathrm{CI})$ smoking pack years in the cigarette smokers was 21.9 (6.8-37.0) pack years. In these subjects, resistin also showed significant inverse relationships with FEV1\% $(r=-0.28 ; p=0.04)$; FEV1/FVC $\%(r=-0.26 ; p=0.04)$; BMI $(r=-0.31$, $p=0.04)$ and HDL cholesterol $(r=-0.38 ; p=$ $0.03)$ and significant positive correlation with HOMAIR $(r=0.27, p=0.03)$. 
Table 1

Characteristics of the subjects. Results are mean (95\% confidence interval)

\begin{tabular}{|c|c|c|c|c|c|}
\hline Parameter & Controls & Acute asthma & Stable asthma & COPD & Smokers \\
\hline Age (years) & $41.4(36.8-47.1)$ & $51.9(45.7-58.1)^{*}$ & $46(39.5-52.5)$ & $68.0(64.6-71.5)^{* *}$ & $37.8(36.2-39.4)$ \\
\hline $\mathrm{BMI}\left(\mathrm{kg} / \mathrm{m}^{2}\right)$ & $26.7(25.0-28.4)$ & $27.9(25.0-30.8)$ & $25.9(23.4-28.4)$ & $21.1(18.7-23.4)$ & $26.9(25.9-28.0)$ \\
\hline Fasting Glucose (mmol/L) & $5.2(4.7-5.7)$ & $5.6(5.1-6.0)$ & $5.2(4.7-5.7)$ & $5.8(5.2-6.4)$ & $5.5(5.4-5.6)$ \\
\hline Fasting Insulin $\mu \mathrm{IU} / \mathrm{ml}$ & $5.3(4.1-6.3)$ & $17.8(10.5-25.2)^{* * * *}$ & $19.6(11.5-27.7)^{* * *}$ & $10.7(5.6-15.7)^{* *}$ & $5.9(5.3-6.4)$ \\
\hline HOMA-IR & $1.3(0.9-1.7)$ & $2.3(1.6-2.9)^{*}$ & $2.3(1.4-3.2)^{* *}$ & $1.6(0.8-2.3)$ & $1.9(1.3-2.6)^{*}$ \\
\hline Total Cholesterol (mmol/L) & $4.3(4.0-4.6)$ & $3.5(3.1-3.8)$ & $3.9(3.5-4.4)$ & $3.5(3.0-4.0)$ & $5.2(5.0-5.4)^{*}$ \\
\hline Triglycerides (mmol/L) & $1.3(1.1-1.5)$ & $1.1(0.9-1.2)$ & $1.6(1.4-1.9)^{*}$ & $1.0(0.8-1.2)$ & $1.6(1.4-1.8)^{*}$ \\
\hline HDL Cholesterol (mmol/L) & $0.9(0.8-1.0)$ & $0.9(0.8-1.1)$ & $0.7(0.6-0.9)$ & $0.9(0.7-1.1)$ & $1.0(1.0-1.1)$ \\
\hline LDL Cholesterol (mmol/L) & $2.8(2.5-3.0)$ & $2.1(1.8-2.3)$ & $2.5(2.1-2.8)$ & $2.1(1.7-2.4))$ & $3.7(3.5-3.9)$ \\
\hline Resistin (ng/mL) & $4.2(3.7-4.8)$ & $6.9(5.1-8.7)^{* *}$ & $4.6(3.0-6.2)^{*}$ & $8.1(3.8-12.4)^{* * *}$ & $8.9(7.0-10.8)^{* *}$ \\
\hline Complement C3 g/L & $0.9(0.8-0.9)$ & $1.5(1.3-1.6)^{* *}$ & $1.7(1.5-1.8)^{* *}$ & $1.4(1.3-1.4)^{* *}$ & $1.4(1.1-1.7)^{* * *}$ \\
\hline Complement $\mathrm{C} 4 \mathrm{~g} / \mathrm{L}$ & $0.3(0.2-0.3)$ & $0.4(0.4-0.5)^{*}$ & $0.4(0.4-0.4)^{*}$ & $0.4(0.3-0.5)^{*}$ & $0.4(0.3-0.5)^{*}$ \\
\hline FEV1\% & Not Done & $53(44-62)$ & $74(62-86)$ & $44(32-57)$ & $90.4(87.7-93.1)$ \\
\hline FEV1 / FVC\% & Not Done & $64(57-72)$ & $85(75-94)$ & $54(43-64)$ & $89.3(88.0-90.7)$ \\
\hline
\end{tabular}

***Difference from control group is significant at the 0.0001 level (2-tailed).

$* *$ Difference from control group is significant at the 0.001 level (2-tailed).

*Difference from control group is significant at the 0.05 level (2-tailed).

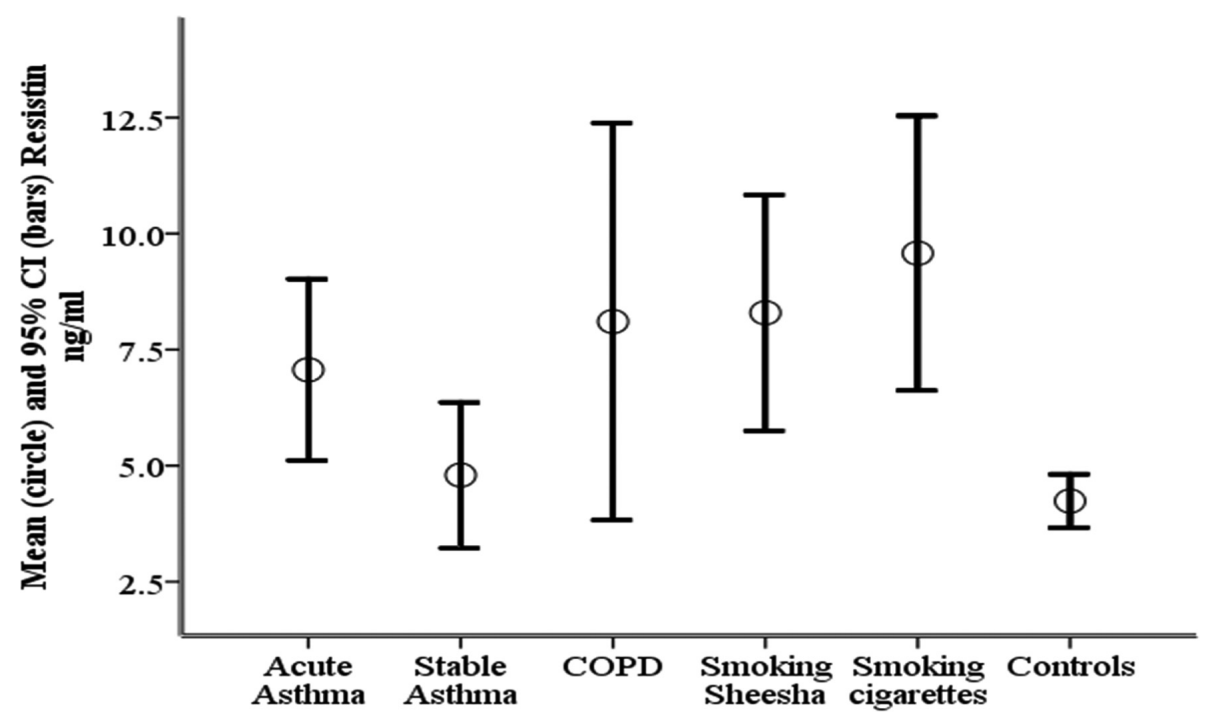

Fig. 1. Error bar plots showing serum resistin in the different groups of subjects studied. The circles in the middle of the error bars represent the mean and the bars represent the $95 \%$ confidence interval (CI). COPD $=$ chronic obstructive pulmonary disease.

\subsection{Regression analyses}

On binary logistic regression with obstructive airways disease (asthma or COPD) as the dependent variable, resistin was significantly associated with inflammatory obstructive airways disease odds ratio (OR) and (95\% confidence interval $(\mathrm{CI})=1.22(1.12-1.33) p=$ 0.001 . However when the confounding effects of age, BMI, glucose, insulin and HOMA-IR were included in the model, the association became insignificant, OR $(95 \% \mathrm{CI})=1.12(0.93-1.31) ; p=0.12$. However, resistin was significantly associated with smoking, OR $(95 \% \mathrm{CI})=1.18(1.10-1.28) ; p<0.0001$ and this as- sociation remained significant when confounding factors (age, BMI, glucose, insulin and HOMA-IR) were included in the model, OR $(95 \% \mathrm{CI})=1.2(1.04-1.42)$.

\section{Discussion}

In this study we have shown that resistin is significantly higher in patients with inflammatory obstructive airways disease compared to control subjects. We have also shown that smoking is associated with higher resistin and inflammatory markers C3, C4. Our finding of higher resistin, C3 and C4 in smokers is novel as 


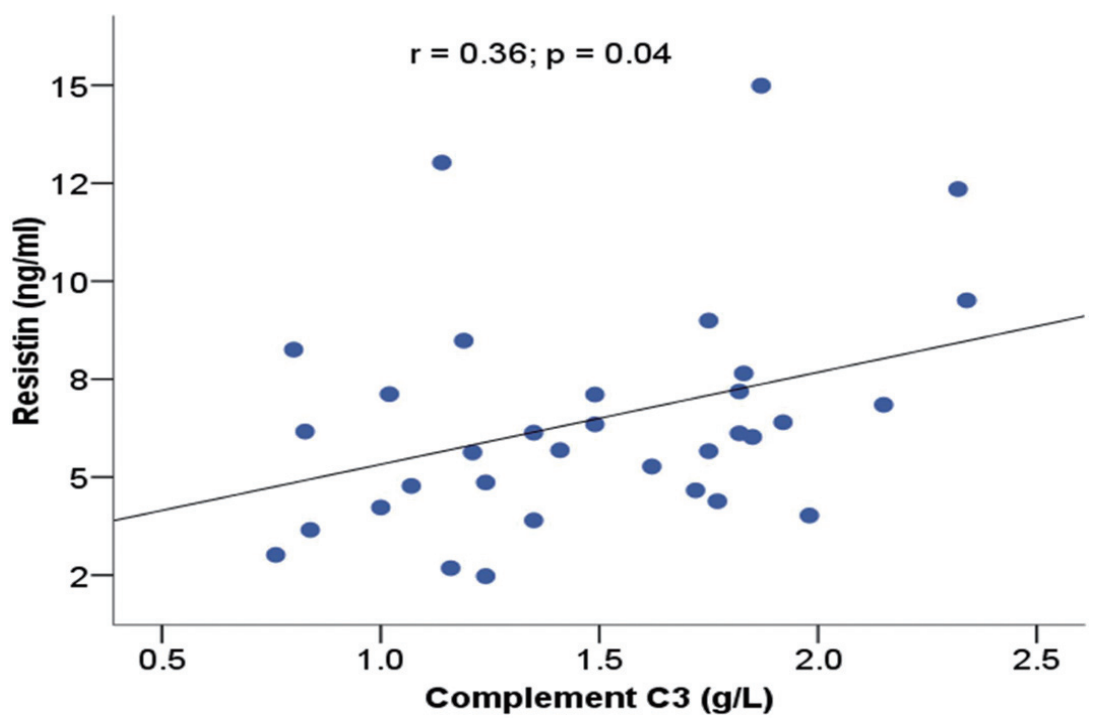

Fig. 2. Scatter plot showing the relationship between resistin and complement C3 in patients with acute asthma exacerbation. Line through the scatter points is regression based fit line showing the trend of the data.

we are not aware of previous studies showing this phenomenon. This finding suggests that smoking produces the same types of effect as inflammatory obstructive airways diseases.

The finding that resistin is increased in subjects with pulmonary inflammation confirms earlier studies [10, 11]. Although, initially identified as a protein, secreted by adipocytes with potential roles in adipose tissue differentiation and insulin resistance, several studies have now confirmed that resistin is associated with inflammation [7-9]. In fact, one of the resistin-like molecules (RELM), RELM $\alpha$ was originally found in inflammatory zones in a murine model of experimental asthma [25]. It is now believed that the predominant producer of resistin in humans are macrophages [26] but whether resistin is simply a marker of inflammation or an active promoter of airway inflammation and even lung remodeling in humans remains a matter of conjecture. There are reports which suggest that resistin plays a role in promoting airway inflammation in a murine animal model [27]. In the study by Mishra et al. [27] intrathecal administration of RELM-beta resulted in dosedependent increased accumulation of macrophages and goblet cell hyperplasia. The correlation of resistin with complement C3 (Fig. 2) supports the fact that the role of resistin in this experimental model of allergic airway inflammation is similar to what occurs in humans. We have also shown that smoking and inflammatory obstructive airways disease share similar responses with regards to resistin, $\mathrm{C} 3$ and $\mathrm{C} 4$. This is not surprising because smoking may affect airway function in similar ways due to its toxic and pro-inflammatory effects [28].

Resistin probably contributes to the higher insulin and HOMA-IR in patients with inflammatory airways disease and smokers. The high insulin levels (Table) in relation to acute asthma exacerbation, chronic stable asthma and COPD could be explained in terms of the effects of intermittent hypoxia (as occurs in acute asthmatics) versus chronic hypoxia (COPD patients) on glucose metabolism. It is known that acute exposure to hypoxia at high altitudes [29-31] results in worsening of glucose tolerance; whereas sustained exposure to hypoxia at high altitude is not associated with persistent abnormalities in glucose homeostasis [30] partly because chronic high altitude exposure stimulates glucose production but increases peripheral insulin sensitivity. Moreover, for a given level and duration of exposure, the systemic and cellular responses that affect glucose homeostasis are more potent with intermittent than with sustained hypoxia [32] which might explain the high and variable levels of insulin (Table) in patients with asthma and COPD. This could also explain the higher HOMA-IR observed in patients with asthma compared to controls and patients with COPD. The observation of significantly higher HOMA-IR in smokers (Table 1) is in agreement with the well known association of smoking with insulin resistance [33]. The correlation of resistin with HOMA-IR in smokers suggests that resistin may be a contributory factor to the insulin resistant state. Apart from the cross-sectional design which can not prove "the cause and effect relationships", the only 
other limitation of this study is that we did not do $a$ priori sample size calculations for detection of differences in HOMA-IR resistin concentrations in the different subgroups. However, a posteriori calculations showed that the power to detect differences between the controls and other subgroups at an alpha error of 0.05 was as high as 0.80 for HOMA-IR and 0.85 for resistin.

In conclusion, this study has confirmed and extended earlier observations that showed resistin to be a marker of pulmonary inflammation. Our results suggest that smoking and conditions with pulmonary inflammation are accompanied by higher resistin which contributes to an insulin resistant state. The higher resistin in smokers is independent of the BMI and degree of insulin resistance and may be a key player linking smoking, insulin resistance and cardiovascular disease.

\section{Acknowledgment}

We thank Mr. Denise Javier and Mrs. Nadia Faraj for their help in recruiting subjects and for technical assistance.

\section{References}

[1] C.M. Steppan, S.T. Bailey, S.T. Bhat et al., The hormone resistin links obesity to diabetes, Nature 409 (2001), 307-312.

[2] L. Patel, A.C. Buckels, I.J. Kinghorn et al., Resistin is expressed in human macrophages and directly regulated by PPAR activators, Biochem Biophys Res Commun 300 (2003), 472-476.

[3] H.-C. Fehmann and J. Heyn, Plasma resistin levels in patients with type 1 and type 2 diabetes mellitus and in healthy controls, Horm Metab Res 34 (2002), 671-673.

[4] J.H. Lee, J.L. Chan, N. Yiannakouris et al., Circulating resistin levels are not associated with obesity or insulin resistance in humans and are not regulated by fasting or leptin administration: cross-sectional and interventional studies in normal, insulin-resistant, and diabetic subjects, J Clin Endocrinol Metab 88 (2003), 4848-4856.

[5] P.G. McTernan, F.M. Fisher, F.M. Valsamakis et al., Resistin and type 2 diabetes: regulation of resistin expression by insulin and rosiglitazone and the effects of recombinant resistin on lipid and glucose metabolism in human differentiated adipocytes, J Clin Endocrinol Metab 88 (2003), 6098-6106.

[6] O.A. Mojiminiyi and N.A. Abdella, Association of resistin with inflammation and insulin resistance in patients with type 2 diabetes mellitus, Scandinavian J of Clinical and Laboratory Investigation 67 (2007), 215-225.

[7] M. Lehrk, M.P. Reilly, S.C. Millington, N. Iqbal, D.J. Rader and M.A. Lazar, An inflammatory cascade leading to hyperreisitinemia in humans, Plos Medicine/ Public Library of Science 1 (2004), e45.
[8] P.G. McTernan, C.M. Kusminski and S. Kumar, Resistin, Current Opinion in Lipidology 17 (2006), 170-175.

[9] S.S. Pang and Y.Y. Le, Role of resistin in inflammation and inflammation-related diseases, Cellular \& Molecular Immunology 3 (2006), 29-34.

[10] J. Larochelle, J. Freiler, J. Dice and J. Hagan, Plasma resistin levels in asthmatics as marker of disease state, J Asthma 44 (2007), 509-513.

[11] A. Mishra, M. Wang, J. Scholtman et al., Resistin-like molecule -beta is an allergen -induced cytokine with inflammatory and remodeling activity in the murine lung, Am J Physio Lung Cell Mol Physiol 293 (2007), L305-L313.

[12] G.K. Shetty, P.A. Economides, E.S. Horton, C.S. Mantzoros and A. Veves, Circulating adiponectin and resistin levels in relation to metabolic factors, in flammatory markers, and vascular reactivity in diabetics patients and subjects at risk of diabetes, Diabetes Care 27 (2004), 2450-2457.

[13] O.A. Mojiminiyi, F. Al Mulla, N.A. Abdella, Which obesity index best explains the link between adipokines, coronary heart disease risk and metabolic abnormalities in type 2 diabetes mellitus? Med Princ Pract 18 (2009), 123-129.

[14] H. Raff and E.D. Bruder, Adiponectin and resistin in the neonatal rat: effects of dexamethosone and hypoxia, Endocrine 29 (2006), 341-344.

[15] P. Trayhurn and I.S. Wood, Adipokines: inflammation and the pleiotropic role of white adipose tissue, British Journal of Nutrition 92 (2004), 347-355.

[16] F.I.E. Najam, A.S.M. Giasuddin and A.H. Shembesh, Complement components (C3, C4) in childhood asthma, Indian J Pediatr 72 (2005), 745-749.

[17] M.M. Marc, P. Korosec, M. Kosnik et al., Complement factors $\mathrm{C} 3 \mathrm{a}, \mathrm{C} 4 \mathrm{a}$, and $\mathrm{C} 5 \mathrm{a}$ in chronic obstructive pulmonary disease and asthma, Am J Respire Cell Mol Boil 31 (2004), 216-219.

[18] E.N. Kosmas, D. Zorpidodou, V. Vassilareas, T. Roussou and S. Michaelides, Decreased C4 complement component serum levels correlate with the degree of emphysema in patients with chronic bronchitis, Chest 112 (1997), 341-347.

[19] National Asthma Education and Prevention Program: Expert Panel Report III: Guidelines for the diagnosis and management of asthma. Bethesda, MD. National Heart, Lung, and Blood Institute, 2007. (NIH publication no. 08-4051). Available from www.nhlbi.nih.gov/guidelines/asthma/asthgdln.htm.

[20] R.A. Pauwels, A.S. Buist, P.M. Calverley, R.C. Jenkins and S.S. Hurd, On the behalf of the Gold Scientific Committee. Global strategies for the diagnosis, management and prevention of chronic obstructive pulmonary disease. NHLBI/ WHO Global Initiative for chronic obstructive lung disease (GOLD) Workshop Summary, Am J Resp Crit Care Med 163 (2001), 1256-1276.

[21] World Health Organisation. Measuring obesity: classification and distribution of anthropometric data. Copenhagen: WHO, 1989. (Nutr UD, EUR/ICP/NUT 125).

[22] Standardization of Spirometry, 1994 Update. American Thoracic Society, Am J Resp Crit Care Med 152 (1995), 11071136.

[23] W.T. Friedewald, R.T. Levy and D.S. Fredrickson, Estimation of the concentration of low density lipoprotein cholesterol in plasma without use of the ultracentrifuge, Clin Chem 18 (1972), 449-502.

[24] Homeostasis model assessment (HOMA) formula using a calculator downloaded from http://www.dtu.ox.ac.uk/index. html?maindoc=/publications/: date accessed May 2008.

[25] G. Raes, L. Brys, B.K. Dahal et al., Macrophage galactosetype C-type lectins as novel markers for alternatively activat- 
ed macrophages elicited by parasitic infections and allergic airway inflammation, J Leukoc Biol 77 (2005), 321-327.

[26] L. Patel, A.C. Buckels, I.J. Kinghorn et al., Resistin is expressed in human macrophages and directly regulated by PPAR gamma activators, Biochem Biophys Res Commun 300 (2003), 472-476.

[27] A. Mishra, M. Wang, J. Schlotman et al., Resistin-like molecule-beta is an allergen-induced cytokine with inflammatory and remodeling activity in the murine lung, American Journal of Physiology - Lung Cellular \& Molecular Physiology 293 (2007), L305-L313.

[28] R.P. Schleimer, Innate Immune Responses and Chronic Obstructive Pulmonary Disease, "Terminator" or "Terminator 2"? The Proceedings of the American Thoracic Society 2 (2005), 342-346.

[29] B. Braun, P.B. Rock, S. Zamudio, Wolfel et al., Women at altitude: short-term exposure to hypoxia and/or $\alpha_{1}$-adrenergic blockade reduces insulin sensitivity, J Appl Physiol 91 (2001), 623-631.

[30] J.J. Larsen, J.M. Hansen, N.V. Olsen, H. Galbo and F. Dela, The effect of altitude hypoxia on glucose homeostasis in men, J Physiol 504 (1997), 241-249.

[31] K.M. Oltmanns, H. Gehring, H. Rudolf et al., Hypoxia causes glucose intolerance in humans, Am J Respir Crit Care Med 169 (2004), 1231-1237.

[32] N.R. Prabhakar and G.K. Kumar, Oxidative stress in the systemic and cellular responses to intermittent hypoxia, Biol Chem 385 (2004), 217-221.

[33] G.K. Reaven and P.S. Tsao, Insulin resistance and compensatory hyperinsulinemia: the key player between cigarette smoking and cardiovascular disease? Journal of the American College of Cardiology 41 (2003), 1044-1047. 


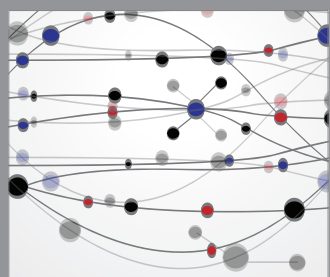

The Scientific World Journal
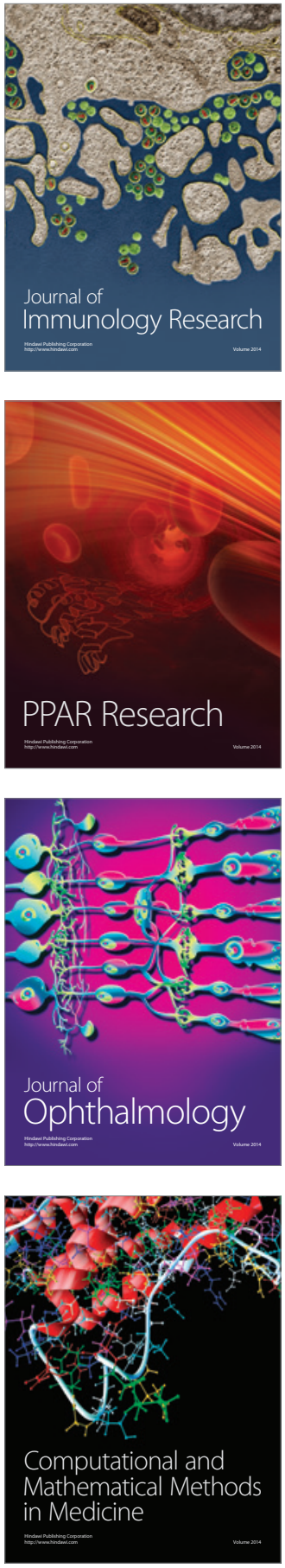

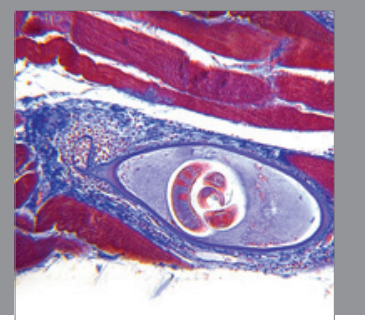

Gastroenterology

Research and Practice
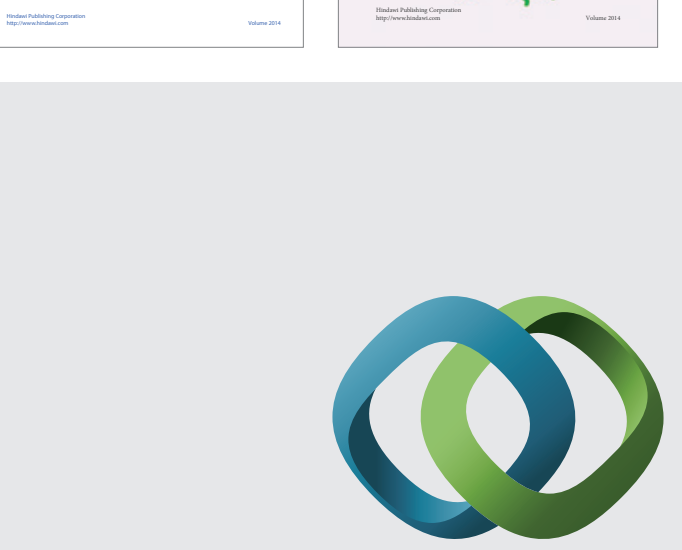

\section{Hindawi}

Submit your manuscripts at

http://www.hindawi.com
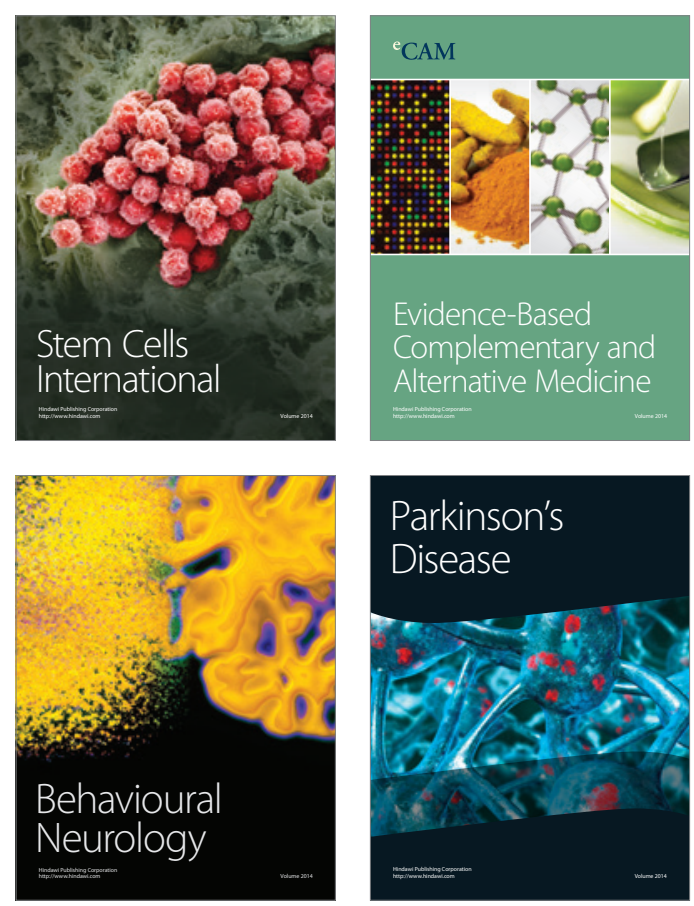

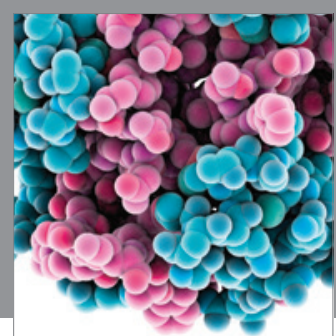

Journal of
Diabetes Research

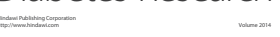

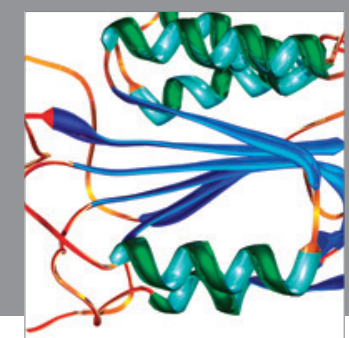

Disease Markers
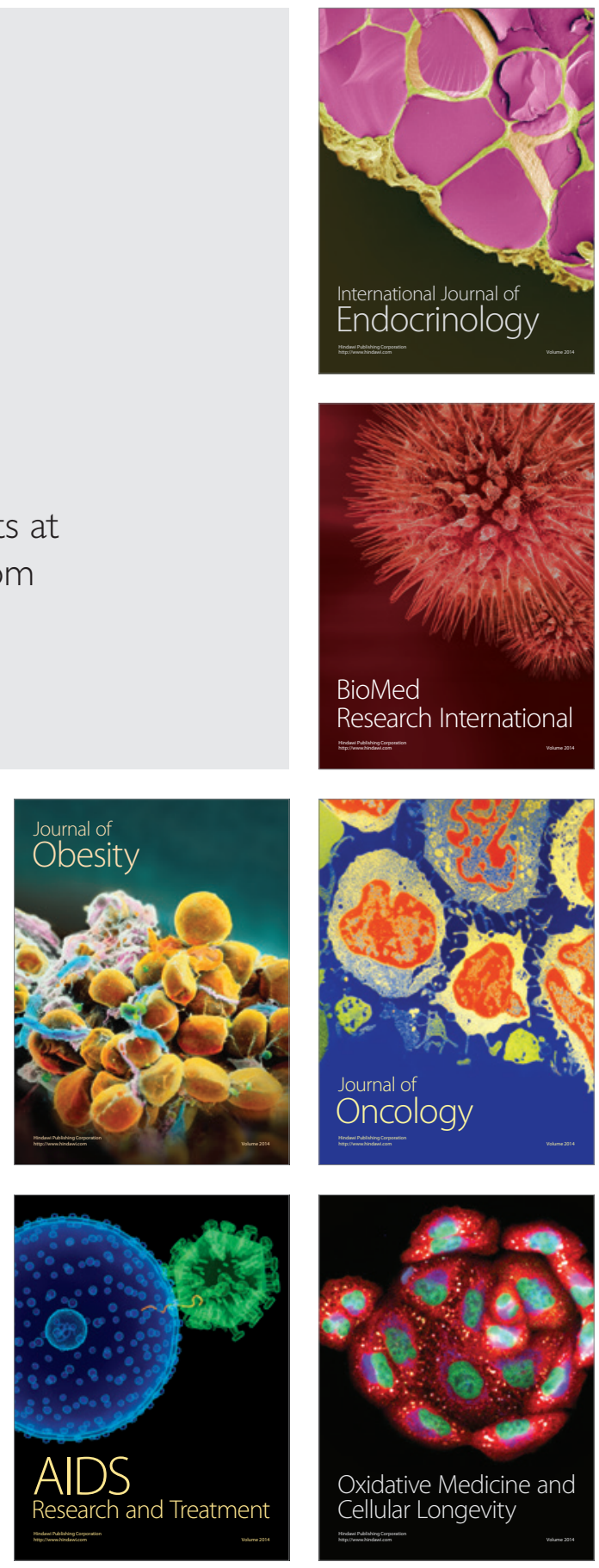